\title{
Delivery of Linear Gene-Editing Systems by Cell-Penetrating Magnetite Vehicles: Synthesis, Characterization and Preliminary In Vitro Testing ${ }^{+}$
}

\author{
Tatiana Beltrán ${ }^{1}$, Javier Cifuentes ${ }^{1}$, Maria Claudia Castellanos ${ }^{1}$, Paola Ruiz ${ }^{1}$, Laura Ellis ${ }^{1}$, \\ David Arango ${ }^{1}$, Carolina Muñoz-Camargo ${ }^{1}$, Luis H. Reyes ${ }^{2, *}$ and Juan C. Cruz ${ }^{1,3, *}$
}

Citation: Beltrán, T.; Cifuentes, J.; Castellanos, M.C.; Ruiz, P.; Ellis, L.; Arango, D.; Muñoz-Camargo, M.; Reyes, L.H.; Cruz, J.C. Delivery of Linear Gene-Editing Systems by Cell-Penetrating Magnetite Vehicles: Synthesis, Characterization and Preliminary In Vitro Testing. Mater. Proc. 2021, 4, 36. https:// doi.org/10.3390/IOCN2020-07927

Published: 11 November 2020

Publisher's Note: MDPI stays neutral with regard to jurisdictional claims in published maps and institutional affiliations.

Copyright: () 2020 by the authors. Licensee MDPI, Basel, Switzerland. This article is an open access article distributed under the terms and conditions of the Creative Commons Attribution (CC BY) license (http://creativecommons.org/licenses /by/4.0/).
1 Department of Biomedical Engineering, Universidad de los Andes, Bogotá, Colombia, Cra. 1E No. 19a-40, Bogotá, CO 111711, USA; tc.beltran@uniandes.edu.co (T.B.); jf.cifuentes10@uniandes.edu.co (J.C.); mc.castellanos10@uniandes.edu.co (M.C.C.); p.ruiz@uniandes.edu.co (P.R.); ld.ellis10@uniandes.edu.co (L.E.); d.arango@uniandes.edu.co (D.A.); c.munoz2016@uniandes.edu.co (C.M.-C.)

2 Department of Chemical and Food Engineering, Universidad de los Andes, Bogotá, Colombia, Cra. 1E No. 19a-40, Bogotá, CO 111711, USA

3 School of Chemical Engineering and Advanced Materials, University of Adelaide, Adelaide 5000, Australia

* Correspondence: 1h.reyes@uniandes.edu.co (L.H.R.); jc.cruz@uniandes.edu.co (J.C.C.)

+ Presented at the 2nd International Online-Conference on Nanomaterials, 15-30 November 2020; Available online: https://iocn2020.sciforum.net/.

\begin{abstract}
One of the main challenges in gene therapy is the transport of genetic material into target cells. This is mainly due to the need for overcoming several obstacles like rapid genetic material degradation by the physiological environment, low endosomal escape, and limited cell uptake. A meaningful way to increase the efficacy of genetic material delivery is to incorporate magnetite nanoparticles to transport biomolecules with high biocompatibility and relative ease of handling. Moreover, magnetite offers the possibility of controlled fate by magnetic fields and excretion as ferritin. This study aims to develop a nanostructured platform for the immobilization and intracellular release of nucleic acids for gene therapy applications. The system also co-immobilized the potent cell-penetrating protein OmpA (outer membrane protein A). The delivery of the conjugated material was first transiently tested in vitro in the presence of reducing agents via spectrofluorimetry. This was achieved by the presence of a reducible linker in the nanoplatform where the fluorophore rhodamine B was conjugated for proof-of-concept purposes. Based on the in vitro results, we decided to deliver this to neuroblastoma and Vero cells to confirm an endosomal escape of about $85 \%$ as calculated by colocalization. Future experiments will be focused on the hybridization of a gene sequence for the expression of the fluorescent protein mCherry. The obtained nanobioconjugate will also be delivered to cells to evaluate transfection efficiencies.
\end{abstract}

Keywords: magnetite; genetic material; nanoparticles; delivery

\section{Introduction}

Currently, it is estimated that genetic alterations or hereditary conditions cause over 10,000 human diseases. A promising approach to treating these diseases is gene therapy, where defective or altered genes are repaired by a normal gene, or nullify/reduce the defective gene expression [1-3]. To develop gene therapy, it is necessary to first identify the affected genes to then transport the replacing or regulating ones to the target tissues. Some of the methods to accomplish this include viral and non-viral vectors, and direct physical manipulation of the target cells $[4,5]$. Vectors require considerable engineering to assure that they can overcome several biological barriers, which often involves interfacing them 
with molecules that interact with such barriers and mediate their trespassing. Effective molecules include polymers, lipids, and translocating protein and peptides [6-8]. Besides their abilities to come across such barriers, vectors should exhibit high biocompatibility, and once they reach the target cells, the capability to escape endosomal entrapment $[9,10]$. Furthermore, they should display pharmacokinetic and pharmacodynamic profiles that assure appropriate time scales and adequate concentration levels.

Within the non-viral vectors, nanocarriers offer some advantages such as safeness, ease of production, customization (in terms of size and topology) and functionalization, large surface-to-volume ratio, and even the possibility of controlling their fate to specific tissues aided by external stimuli [5,11-13]. Nanotechnology has reached a stage of significant maturity, and now it makes a large arsenal of platforms available to transport numerous types of cargoes. As a result, it enables the effective delivery of enzymes, therapeutic proteins, polysaccharides, and even DNA/RNA. One nanostructured material that has emerged as a versatile workhorse for biomedical applications is magnetite, which is well suited for cell imaging, biological separation, localized heat dissipation, and drug and nucleic acid transport [14-16]. Despite the important advances in magnetite-based vehicles, a major challenge is to overcome endosomal entrapment upon cellular uptake. We have been addressing this major hurdle by interfacing magnetite with cell-penetrating peptides and proteins with remarkable results [17-22]. Here, we selected magnetite functionalized with the translocating and endosome-escaping protein OmpA as a nanocarrier to deliver nucleic acids. To accomplish this, magnetite obtained via co-precipitation was functionalized with an organosilane followed by a surface spacer (polyether amine (PEA) or polyethylene glycol (PEG)) and 3-[(2-aminoethyl)dithio]propionic acid (AEDP), a molecule with a reducible disulfide linker to which a thiolated DNA tag, complementary with a linear sequence of the delivery vector, will be conjugated. Finally, OmpA [19] was coimmobilized directly onto the silanized particles or the PEA/PEG-AEDP-modified magnetite nanoparticles. Once the OmpA-magnetite-AEDP DNA or the OmpA-magnetitePEA/PEG-AEDP DNA nanobioconjugates reach the intracellular space, the disulfide bond is reduced, and the genetic material is delivered. To test the potential for delivery, the model fluorophore molecule rhodamine B was conjugated to the OmpA-magnetiteAEDP or OmpA-magnetite-PEA/PEG-AEDP nanobioconjugates. The delivery of rhodamine $B$ was first tested in vitro with the aid of reducing agents. The delivery was transiently tracked with the assistance of spectrofluorimetry. Later, we decided to deliver to neuroblastoma and Vero cells to confirm the effective endosomal escape.

\section{Materials and Methods}

\subsection{Synthesis and Functionalization of Magnetite Nanoparticles}

The nanoparticles were obtained by the chemical co-precipitation method, in which solutions of iron chloride (1l) $(0.1 \mathrm{M})$ and iron chloride (1ll) $(0.2 \mathrm{M})$ were mixed in the presence of $1 \mathrm{M}$ sodium hydroxide. The mixture was then heated up to $80^{\circ} \mathrm{C}$. Further details of the synthesis can be consulted elsewhere $[19,21]$. The as-synthesized magnetite nanoparticles were then put under constant mechanical stirring at $300 \mathrm{rpm}$, followed by the addition of $1000 \mu \mathrm{L}$ of tetramethylammonium hydroxide (TMAH), $50 \mu \mathrm{L}$ of glacial acetic acid, and $1000 \mu \mathrm{L}$ of the organosilane 3-amino propyl triethoxy-silane (APTES). The mixture was then heated up to $60^{\circ} \mathrm{C}$ and left to react for $1 \mathrm{~h}$. Next, two types of functionalization were performed on the silanized nanoparticles, which exhibit free amine groups on their surface. On the one hand, the amine-amine conjugations to form imine bonds aided by glutaraldehyde, which were intended for immobilizing PEA and OmpA molecules, reacted for $24 \mathrm{~h}$ to form imine bonds. On the other hand, amine-carboxyl ones with the aid of $\mathrm{N}$-[3-(dimethylamino propyl]-N'-ethyl carbodiimide (EDC) and N-hydroxysuccinimide (NHS) to form amide bonds. In this case, the conjugation was for immobilizing PEG, AEDP, and rhodamine B molecules. In both cases, the conjugation reaction was carried out for $24 \mathrm{~h}$ at room temperature and under vigorous agitation. After each 
functionalization, the obtained nanobioconjugates were washed several times with MilliQ water aided by magnetic separation between washes.

\subsection{Nanoparticle Characterization}

Particle size distribution for magnetite nanoparticles and nanobioconjugates was performed with dynamic light scattering (DLS, Zeta-Sizer Nano-ZS). The effectiveness of immobilization was confirmed by Fourier transform infrared spectroscopy (FTIR) using a Bruker Alpha II FTIR Eco-ATR. Spectra were collected in the range of $4000-400 \mathrm{~cm}^{-1}$ with a spectral resolution of $2 \mathrm{~cm}^{-1}$. TGA (thermogravimetric analysis) was carried out by ramping up a $10 \mathrm{mg}$ sample temperature at a rate of $10{ }^{\circ} \mathrm{C} / \mathrm{min}$ from 25 to $800{ }^{\circ} \mathrm{C}$ in a simultaneous TGA/DSC (differential scanning calorimetry) instrument. A transmission electron microscope (TEM) Tecnai F30, at a resolution of $134 \mathrm{eV}$ and a reference energy of $5.9 \mathrm{keV}$, was used to analyze the magnetite.

\subsection{Delivery Test}

The testing of the disulfide linker present in the OmpA-magnetite-AEDP (vehicle A), OmpA-magnetite-PEG-AEDP (vehicle B), and OmpA-magnetite-PEA-AEDP (vehicle $C$ ) nanobioconjugates was conducted after the conjugation of rhodamine $B$ to the amine terminal of each of them. The delivery of rhodamine B was induced by reduction in the disulfide bond in the presence of dithiothreitol (DTT), $10 \mathrm{mM}$. Two sets of experiments were conducted independently, in the presence (every $30 \mathrm{~min}$ ) and the absence of ultrasonic bath sonication. The delivery was followed for $8 \mathrm{~h}$ at $37^{\circ} \mathrm{C}$ and $210 \mathrm{rpm}$ by taking supernatant aliquots after 1,2,4, and $8 \mathrm{~h}$. The sample intensity was analyzed in a spectrofluorometer with excitation at $550 \mathrm{~nm}$ and emission at $574 \mathrm{~nm}$ (Flourolog 4, Horiba Scientific).

\subsection{Cell Translocation and Endosomal Escape}

Cell translocation and endosomal escape of the vehicles A and B were evaluated in neuroblastoma (ATCC ${ }^{\circledR S H}-S Y 5 Y$ ) cells, and vehicle $\mathrm{C}$ was assessed in Vero cells (ATCC ${ }^{\circledR}$ CCL-81). The cells were cultured in DMEM (Dulbecco's modified Eagle's medium) media supplemented with $10 \%$ FBS (fetal bovine serum) and $1 \% \mathrm{P} / \mathrm{S}$, at $37{ }^{\circ} \mathrm{C}$ and $5 \% \mathrm{CO}_{2}$, to promote adhesion in a glass slide. The cells were exposed to nanobioconjugates, and then the cells were washed and exposed to DMEM solution with DAPI (4',6diamidino-2-phenylindole, Thermo Fisher, Waltham, MA, USA) and LysoTracker Green DND-26 (Thermo Fisher, Waltham, MA, USA). The cell translocation and endosomal escape of the vehicles were evaluated by a decrease in the fluorescence intensity and colocalization between vehicles with immobilized rhodamine B and LysoTracker Green DND26 after internalization. The vehicles were delivered to the cells in a ratio of 1:100 in $1 \mathrm{~mL}$ of RPMI and were incubated for two hours at $37^{\circ} \mathrm{C}$ and $5 \% \mathrm{CO}_{2}$. Finally, the images obtained were analyzed in ImageJ and Fiji.

\section{Results and Discussion}

To investigate the role of the surface spacer, we prepared three different nanobioconjugates. The first one was produced by directly conjugating AEDP to the surface of the silanized nanoparticles, called vehicle A (OmpA-magnetite-AEDP) (Figure 1a), the second one, called vehicle B, contained PEG (OmpA-magnetite-PEG-AEDP) (Figure 1b) before AEDP, and the third one, called vehicle C, with PEA (OmpA-magnetite-PEA-AEDP) (Figure 1c). 
(a)

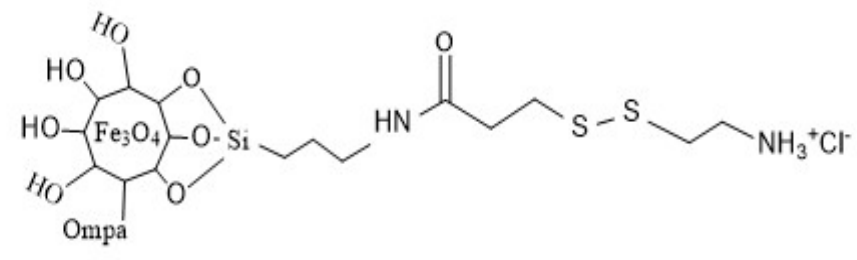

(b)

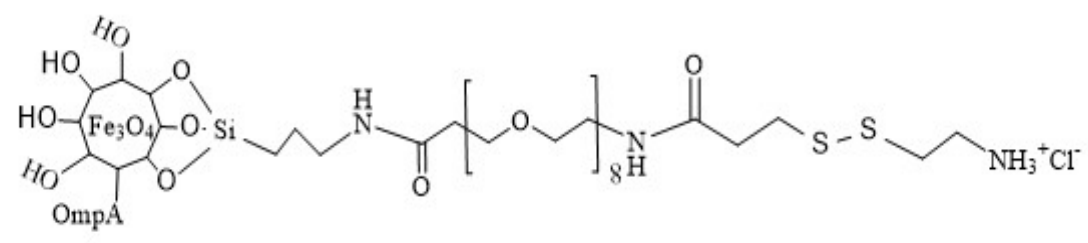

(c) $\mathrm{r}$

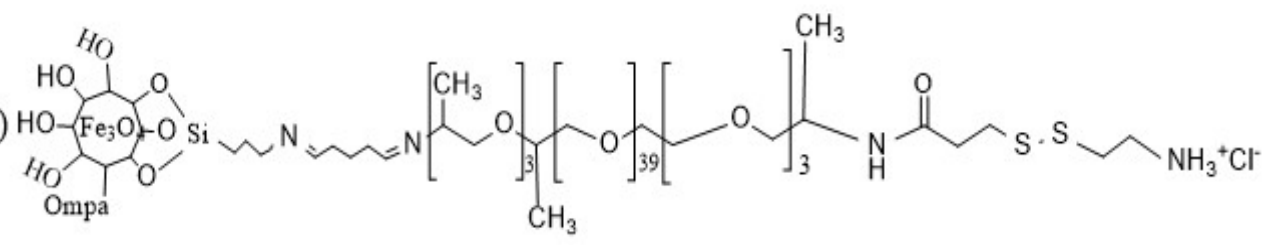

Figure 1. Schematics of the prepared nanobioconjugates as follows: (a) OmpA-magnetite-3-[(2aminoethyl)dithio]propionic acid (AEDP) (Vehicle A), (b) OmpA-magnetite-polyethylene glycol (PEG)-AEDP (Vehicle B), (c) OmpA-magnetite-polyether amine (PEA)-AEDP (Vehicle C).

The nanobioconjugates were characterized by FTIR (Figure 2A), DLS (Figure 2B) and TEM (Figure 2C). Regarding FTIR, without immobilizing the nanoparticles we found iron oxide (Fe-O) vibrations at $610 \mathrm{~cm}^{-1}[23,24]$. After silanization with APTES, we identified the Si-O stretching vibrations at $1047 \mathrm{~cm}^{-1}$, the $\mathrm{C}-\mathrm{H}$ bending vibrations at $1307 \mathrm{~cm}^{-1}$, and the $\mathrm{N}-\mathrm{H}$ bending vibrations $1641 \mathrm{~cm}^{-1}$. After immobilizing the AEDP, we found the $\mathrm{C}-\mathrm{O}$ stretching vibrations at $1769 \mathrm{~cm}^{-1}$, and the S-S vibrations at $623 \mathrm{~cm}^{-1}$ [24,25]. Additionally, the $\mathrm{C}-\mathrm{O}-\mathrm{H}$ stretching vibrations at $1113 \mathrm{~cm}^{-1}$ and the $\mathrm{C}-\mathrm{H}$ bending vibrations at $1457 \mathrm{~cm}^{-1}$ confirmed the presence of PEG [22], and we identified amide I and the amide II at 1641 $\mathrm{cm}^{-1}$ and $1519 \mathrm{~cm}^{-1}$, which can be attributed to the presence of immobilized OmpA.

The magnetite nanoparticles exhibited a hydrodynamic diameter of $137 \mathrm{~nm}$ with a polydispersity index of 0.216 , while vehicles A and B showed hydrodynamic diameters of $188 \mathrm{~nm}$ and $194 \mathrm{~nm}$ with polydispersity indexes of 0.234 and 0.253 , respectively. TGA analysis confirmed a conjugation efficiency of PEG of about $1.5 \%$ and PEA of about $5 \%$, while for OmpA the efficiency approached 1.5\%. TEM allowed us to confirm the nanoparticles' typical spherical morphology and an average diameter for individual particles of about $15-18 \mathrm{~nm}$.

The release of rhodamine B was then studied with the spectrofluorometer, showing the disulfide bond reduction by the presence of DTT in the medium. This simulated the reducing conditions of the intracellular space. As time progressed, the release of rhodamine B was evidenced by an increase in the fluorescence intensity of the medium. After this, we decided to test the developed vehicles in the neuroblastoma cell line. 
(A)

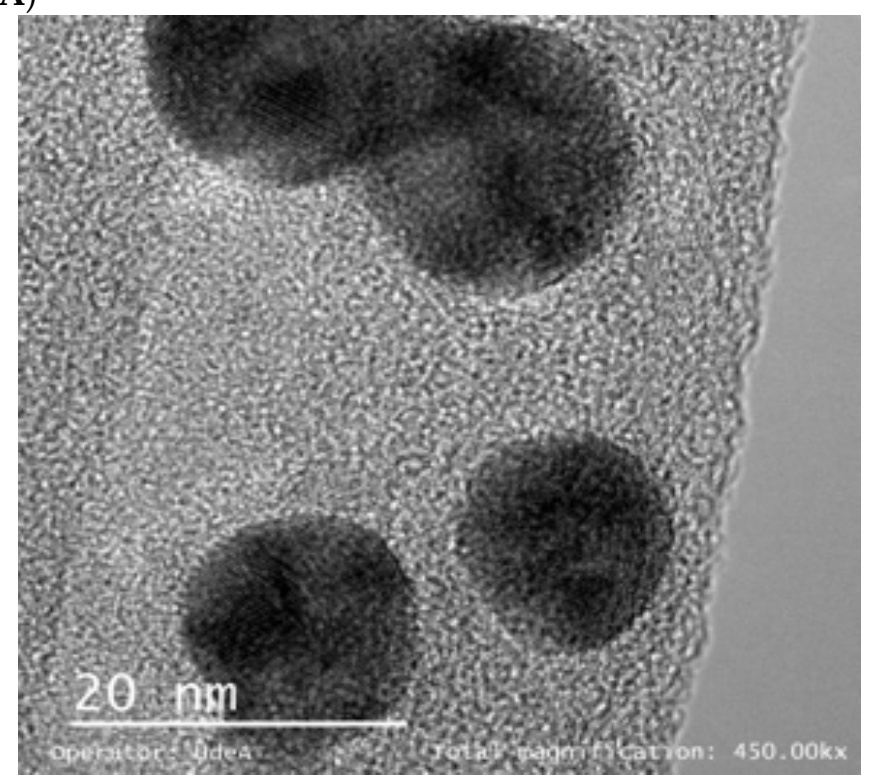

(C)
(B)

Figure 2. Characterization of vehicles A and B. (A) Fourier transform infrared spectroscopy (FTIR) spectra were used to evaluate the effectiveness of the synthesis and each immobilization step for magnetite (green), vehicle A (orange) and vehicle C (blue). (B) Nanoparticles size distribution via dynamic light scattering (DLS), (C) transmission electron microscope (TEM) micrograph of magnetite nanoparticles with diameters ranging from $15.8 \mathrm{~nm}$ to $17.3 \mathrm{~nm}$.

Our results indicated that the vehicles translocated the cell membrane without affecting their viability (data not shown). From colocalization analyses in neuroblastoma (ATCC ${ }^{\circledR S H}$-SY5Y) cells between the nanobioconjugates and the endosome-labeling probe LysoTracker Green, we concluded that after two hours of exposure, vehicle A showed an endosomal escape of $67.1 \%$ (Figure 3A), vehicle B of 70.7\% (Figure 3B), and vehicle C of $85 \%$ (Figure 3 C). In addition, colocalization analyses with Vero (ATCC ${ }^{\circledR}$ CCL-81) cells for vehicle $\mathrm{C}$ led to an endosomal escape of $31.4 \%$. This corroborates that the proposed strategy for internalization and subsequent escape appears robust enough for further transfection experiments where the genetic material should reach the cytosol.
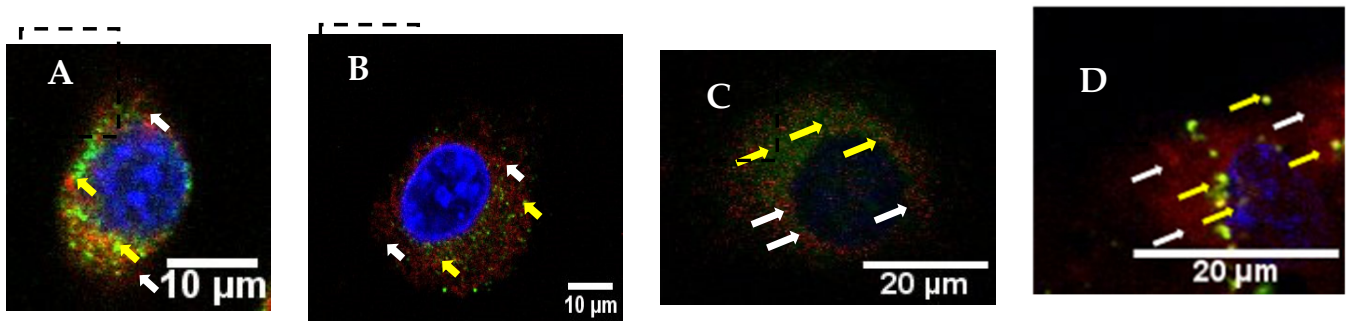

Figure 3. Cell translocation and endosomal escape. (A) Confocal microscopy images of effective cellular and nuclear internalization of vehicle A in neuroblastoma cells after two hours. (B) Confocal microscopy images of effective cellular and nuclear internalization of vehicle B in neuroblastoma cells after two hours. (C) Confocal microscopy images of effective 
cellular and nuclear internalization of vehicle $C$ in neuroblastoma cells after two hours. (D) Confocal microscopy images of effective cellular and nuclear internalization of vehicle $\mathrm{C}$ in Vero cells after half an hour. Yellow arrows point to endosomal compartments where the nanobioconjugates remained trapped, while the white ones point to nanobioconjugates that likely escaped the endocytic trafficking routes.

\section{Conclusions}

The need for more potent vehicles for the delivery of gene therapies has propelled the search for novel viral and non-viral platforms. Due to biosafety issues, non-viral vehicles and particularly nanostructured materials emerge as attractive alternatives. The past two decades have seen the development of a large arsenal of nanomaterials, some of which can be easily synthesized at a relatively low cost. One attractive nanoplatform is magnetite nanoparticles (MNPs) due to the well-established and optimized protocols for their synthesis and functionalization as well as properties such as high biocompatibility and superior magnetic responsiveness. Despite their potential for drug delivery applications, MNPs penetration can be improved further by interfacing them with cell translocation proteins and peptides. Over the past three years, we started a research program to advance in such a conceptual direction by immobilizing, on MNPs, the antimicrobial peptide BUF-II and the translocating protein OmpA. Due to their remarkable endosomal escape abilities, here we decided to explore the potential application of these cell-penetrating agents in the delivery of genetic material. To accomplish this, we successfully synthesized and characterized (via TGA, TEM and DLS) three nanovehicles where the active agent (i.e., OmpA) is co-immobilized with a reducible linker system (based on a disulfide bond) on MNPs. We explored the potential benefits of immobilizing the linker on MNPs modified with two polymeric surface spacers. Delivery in vitro confirmed the suitability of the linker and the spacers for high delivery levels, and they continued with cell delivery. The results confirmed endosomal escape above $60 \%$ as calculated from colocalization experiments in a confocal microscope.

\section{Future Work}

The next steps involve the conjugation of a thiolated DNA sequence of $65 \mathrm{bp}$ length that is complementary to a non-encoding region of a CRISPR/Cas9 plasmid for gene edition in some innate inborn error of metabolism diseases. In this regard, we expect to linearize the plasmid and hybridize it for delivery to diseased cells.

Author Contributions: conceptualization, J.C.C., C.M.-C. and L.H.R.; methodology, data curation, and data analysis T.B., J.C., M.C.C., P.R., L.E. and D.A.; formal analysis and investigation, T.B., J.C., M.C.C., P.R., L.E. and D.A.; validation, J.C.C., C.M.-C. and L.H.R.; writing-original draft preparation, T.B.; writing - review and editing, J.C.C., C.M.-C. and L.H.R.; supervision, J.C.C., C.M.-C. and L.H.R. All authors have read and agreed to the published version of the manuscript.

Funding: This work was funded by the Colombian Ministry of Science, Technology, and Innovation (Minciencias) Grants 689-2018, and ID 120380763212-PPTA \# 8352.

Acknowledgments: The authors would like to thank the Department of Biomedical Engineering, Food and Chemical engineering and the Department of Electrical \& Electronics Engineering at Universidad de los Andes for the financial and technical support.

Conflicts of Interest: The authors declare no conflict of interest.

\section{References}

1. Weng, Y.; Huang, Q.; Li, C.; Yang, Y.; Wang, X.; Yu, J.; Huang, Y.; Liang, X.J. Improved Nucleic Acid Therapy with Advanced Nanoscale Biotechnology. Mol. Ther. Nucleic Acids 2020, 19, 581-601.

2. Verma, I.M.; Naldini, L.; Kafri, T.; Miyoshi, H.; Takahashi, M.; Blömer, U.; Somia, N.; Wang, L.; Gage, F.H. Gene Therapy: Promises, Problems and Prospects. In Genes and Resistance to Disease; Springer: Berlin/Heidelberg, Germany, 2000 ; pp. $147-157$.

3. Guo, Z.S.; Li, Q.; Bartlett, D.L.; Yang, J.Y.; Fang, B. Gene transfer: The challenge of regulated gene expression. Trends Mol. Med. 2008, 14, 410-418, doi:10.1016/j.molmed.2008.07.003. 
4. Alsaggar M. and Liu, D. "Physical Methods for Gene Transfer," in Nonviral Vectors for Gene Therapy Physical Methods and Medical Translation, vol. 89, E. W. Leaf Huang, Dexi Liu, Ed. ScienceDirect: Athens, GA, USA: 2015, pp. 1-24.

5. Schillinger, U.; Brill, T.; Rudolph, C.; Huth, S.; Gersting, S.; Krötz, F.; Hirschberger, J.; Bergemann, C.; Plank, C. Advances in magnetofection-Magnetically guided nucleic acid delivery. J. Magn. Magn. Mater. 2005, 293, 501-508, doi:10.1016/j.jmmm.2005.01.032.

6. Ambjörnsson, T.; Apell, S.P.; Konkoli, Z.; Di Marzio, E.A.; Kasianowicz, J.J. Charged polymer membrane translocation. J. Chem. Phys. 2002, 117, 4063-4073, doi:10.1063/1.1486208.

7. Cruz, J.; Mihailescu, M.; Wiedman, G.; Herman, K.; Searson, P.C.; Wimley, W.C.; Hristova, K. A membrane-translocating peptide penetrates into bilayers without significant bilayer perturbations. Biophys. J. 2013, 104, 2419-2428, doi:10.1016/j.bpj.2013.04.043.

8. Bevers, E.M.; Comfurius, P.; Dekkers, D.W.C.; Zwaal, R.F.A. Lipid translocation across the plasma membrane of mammalian cells. Biochim. Biophys. Acta Mol. Cell Biol. Lipids 1999, 1439, 317-330, doi:10.1016/S1388-1981(99)00110-9.

9. Barua, S.; Mitragotri, S. Challenges associated with penetration of nanoparticles across cell and tissue barriers: A review of current status and future prospects. Nano Today 2014, 9, 223-243, doi:10.1016/j.nantod.2014.04.008.

10. Phillips, A.J. The challenge of gene therapy and DNA delivery. J. Pharm. Pharmacol. 2001, 53, 1169-1174, doi:10.1211/0022357011776603.

11. Plank, C.; Scherer, F.; Schillinger, U.; Bergemann, C.; Anton, M. Magnetofection: Enhancing and targeting gene delivery with superparamagnetic nanoparticles and magnetic fields. J. Liposome Res. 2003, 13, 29-32.

12. Krötz, F.; Sohn, H.-Y.; Gloe, T.; Plank, C.; Pohl, U. Magnetofection Potentiates Gene Delivery to Cultured Endothelial Cells. J. Vasc. Res. 2003, 40, 425-434, doi:10.1159/000073901.

13. Gersting, S.W.; Schillinger, U.; Lausier, J.; Nicklaus, P.; Rudolph, C.; Plank, C.; Reinhardt, D.; Rosenecker, J. Gene delivery to respiratory epithelial cells by magnetofection. J. Gene Med. 2004, 6, 913-922, doi:10.1002/jgm.569.

14. Kratz, H.; Mohtashamdolatshahi, A.; Eberbeck, D.; Kosch, O.; Hauptmann, R.; Wiekhorst, F.; Taupitz, M.; Hamm, B.; Schnorr, J. MPI Phantom Study with A High-Performing Multicore Tracer Made by Coprecipitation. Nanomaterials 2019, 9, 1466, doi:10.3390/nano9101466.

15. Chanana, M.; Jahn, S.; Georgieva, R.; Lutz, J.-F.; Bäumler, H.; Wang, D. Fabrication of Colloidal Stable, Thermosensitive, and Biocompatible Magnetite Nanoparticles and Study of Their Reversible Agglomeration in Aqueous Milieu. Chem. Mater. 2009, 21, 1906-1914, doi:10.1021/cm900126r.

16. Pan, B.F.; Gao, F.; Gu, H.C. Dendrimer modified magnetite nanoparticles for protein immobilization. J. Colloid Interface Sci. 2005, 284, 1-6, doi:10.1016/j.jcis.2004.09.073.

17. Ramírez-Acosta, C.M.; Cifuentes, J.; Cruz, J.C.; Reyes, L.H. Patchy Core/Shell, Magnetite/Silver Nanoparticles via Green and Facile Synthesis: Routes to Assure Biocompatibility. Nanomaterials 2020, 10, 1857, doi:10.3390/nano10091857.

18. Ramírez-Acosta, C.M.; Cifuentes, J.; Castellanos, M.C.; Moreno, R.J.; Muñoz-Camargo, C.; Cruz, J.C.; Reyes, L.H. PHResponsive, Cell-Penetrating, Core/Shell Magnetite/Silver Nanoparticles for the Delivery of Plasmids: Preparation, Characterization, and Preliminary In Vitro Evaluation. Pharmaceutics 2020, 12, 561, doi:10.3390/pharmaceutics12060561.

19. Lopez-Barbosa, N.; Suárez-Arnedo, A.; Cifuentes, J.; Gonzalez Barrios, A.F.; Silvera Batista, C.A.; Osma, J.F.; Muñoz-Camargo, C.; Cruz, J.C. Magnetite-OmpA Nanobioconjugates as Cell-Penetrating Vehicles with Endosomal Escape Abilities. ACS Biomater. Sci. Eng. 2019, 6, 415-424, doi:10.1021/acsbiomaterials.9b01214.

20. Lopez-Barbosa, N.; Garcia, J.G.; Cifuentes, J.; Castro, L.M.; Vargas, F.; Ostos, C.; Cardona-Gomez, G.P.; Hernandez, A.M.; Cruz, J.C. Multifunctional magnetite nanoparticles to enable delivery of siRNA for the potential treatment of Alzheimer's. Drug Deliv. 2020, 27, 864-875, doi:10.1080/10717544.2020.1775724.

21. Cuellar, M.; Cifuentes, J.; Perez, J.; Suarez-Arnedo, A.; Serna, J.; Groot, H.; Muñoz-Camargo, C.; Cruz, J. Novel BUF2-magnetite nanobioconjugates with cell-penetrating abilities. Int. J. Nanomed. 2018, 13, 8087-8094, doi:10.2147/IJN.S188074.

22. Perez, J.; Cifuentes, J.; Cuellar, M.; Suarez-Arnedo, A.; Cruz, J.C.; Muñoz-Camargo, C. Cell-penetrating and antibacterial BUFII nanobioconjugates: Enhanced potency via immobilization on polyetheramine-modified magnetite nanoparticles. Int. J. Nanomed. 2019, 14, 8483-8497, doi:10.2147/IJN.S224286.

23. Rudolph, M.; Erler, J.; Peuker, U.A. A TGA-FTIR perspective of fatty acid adsorbed on magnetite nanoparticles-Decomposition steps and magnetite reduction. Colloids Surf. A Physicochem. Eng. Asp. 2012, 397, 16-23, doi:10.1016/j.colsurfa.2012.01.020.

24. Habibi, N. Preparation of biocompatible magnetite-carboxymethyl cellulose nanocomposite: Characterization of nanocomposite by FTIR, XRD, FESEM and TEM. Spectrochim. Acta Part A Mol. Biomol. Spectrosc. 2014, 131, 55-58, doi:10.1016/j.saa.2014.04.039.

25. Masnabadi, N.; Ghasemi, M.H.; Beyki, M.H.; Sadeghinia, M. Oxidative dimerization of thiols to disulfide using recyclable magnetic nanoparticles. Res. Chem. Intermed. 2017, 43, 1609-1618, doi:10.1007/s11164-016-2718-1. 